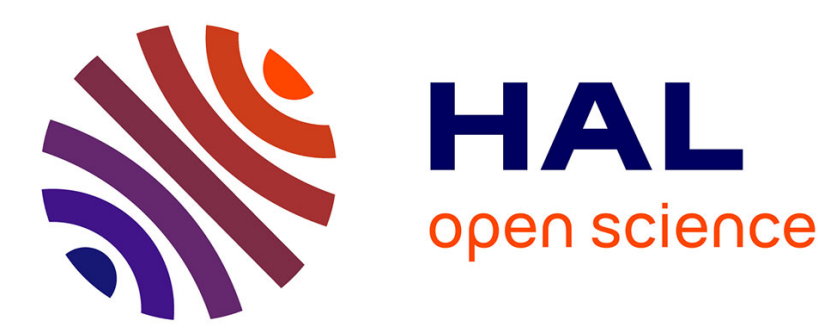

\title{
A Comprehensive Analysis of the Newsvendor Model with Unreliable Supply
}

Yacine Rekik, Evren Sahin, Yves Dallery

\section{To cite this version:}

Yacine Rekik, Evren Sahin, Yves Dallery. A Comprehensive Analysis of the Newsvendor Model with Unreliable Supply. OR Spectrum, 2007, 29 (2), pp.207-233. hal-00393874

\section{HAL Id: hal-00393874 \\ https://hal.science/hal-00393874}

Submitted on 13 Jul 2009

HAL is a multi-disciplinary open access archive for the deposit and dissemination of scientific research documents, whether they are published or not. The documents may come from teaching and research institutions in France or abroad, or from public or private research centers.
L'archive ouverte pluridisciplinaire HAL, est destinée au dépôt et à la diffusion de documents scientifiques de niveau recherche, publiés ou non, émanant des établissements d'enseignement et de recherche français ou étrangers, des laboratoires publics ou privés. 
myjournal manuscript No.

(will be inserted by the editor)

\title{
A Comprehensive Analysis of the Newsvendor Model with Unreliable Supply
}

\author{
Yacine Rekik, Evren Sahin, Yves Dallery \\ Laboratoire Génie Industriel, Ecole Centrale Paris, Grande Voie des Vignes, 92295
}

Chatenay Malabry Cedex, France

The date of receipt and acceptance will be inserted by the editor

\begin{abstract}
A single-period, uncertain demand inventory model is analyzed under the assumption that the quantity ordered (produced) is a random variable. We first conduct a comprehensive analysis of the well known single period production/inventory model with random yield. Then, we extend some of the results existing in literature: our main contribution is to show that earlier results are only valid for a certain range of system parameters. Under the hypothesis that demand and the error in the quantity received from supplier are uniformly distributed, closed-form analytical solutions are obtained for all values of parameters. An analysis under normally distributed demand and error is also provided. The paper ends with an analysis of the benefit achieved by eliminating errors.
\end{abstract}

Keywords: Supply Chain Management, Random Yield, Newsvendor

\section{Introduction}

The classical one-period inventory problem has played an important role for many years in both the theory and applications of inventory control (Silver, Pyke and Peterson (1998) and Khouja (1999)). One of the underlying assumptions in the formulation of the Newsvendor model is that the quantity available to satisfy demand matches the quantity requisitioned from supplier. In the context of inventory systems, the difference between these two quantities stems either from unreliabilities of the supply system or from internal inefficiencies such as misplaced items, perishment or internal theft. A supply system is said to be reliable when the quantity of goods effectively delivered by supplier corresponds exactly to the ordered quantity. The unreliability of the supply system may stem from: $i$ ) Delivery errors or supplier 
frauds defined as losses happening when suppliers deliver fewer goods than ordered; and $i i$ ) Theft during transportation between the vendor and the buyer. For a detailed analysis of potential sources of errors, the reader is referred to Sahin (2004). In production systems, uncertainty in production yield is also a common phenomenon observed in many processes such as electronic fabrication and assembly, and discrete parts manufacturing processes.

The literature in the area of random yield is sparse. The earliest model of a random supply inventory model with random demand was developed by Karlin (1958). This is followed by Shih (1980), Noori and Keller (1986), and Lee and Yano (1988), among many others. Karlin (1958) assumed that the only decision available is whether to order, and that if an order is placed, a random quantity is delivered. He shows that if the inventory holding and shortage cost functions are convex increasing in their respective argument, then there is a single critical initial on-hand inventory below which an order should be placed, otherwise it is optimal not to order. Shih (1980) assumes that inventory holding and shortage costs are linear and that the distribution of the fraction defective is invariant with the production level. He shows that the optimal production/order quantity can be found using a variant of the Newsvendor model. For the problem considered by Shih (1980), Noori and Keller (1986) provide closed form solutions for the optimal order quantity for uniform and exponential demand distributions and for various distributions of the quantity received. Gerchak, Parlar and Vickson (1986) obtain the same result for the profit maximization objective. They assume continuous demand and yield and they consider a model with initial stock. They show that there is a critical level of initial stock above which no order will be placed, and this level is the same as the certain yield case. They show that when initial stock is below that critical level, the expected yield corresponding to the amount ordered will in general not be simply equal to difference. Ehrhardt and Taube (1987) show that when the replenishment quantity is a random fraction of the amount ordered, an optimal single-period ordering policy can be found with a simple generalization of the traditional Newsvendor result. They also show that a simple scaling-up heuristic is an effective approximation to optimal performance. The heuristic computes an order size by starting with the order size that would be optimal with deterministic replenishment, and dividing it by the expected value of the replenishment yield fraction. They propose analytic results for the case of uniformly distributed demand. A significant theoretical contribution was made by Henig and Gerchak (1990), who discuss single and multi-period models with more general assumptions about the random replenishment distribution and the cost structure. They prove that for a single-period model there exists an optimal order point that is independent of replenishment randomness. For an extensive literature on many other variants of the lot sizing problem with random yields, the reader is referred to Yano and Lee (1995). 
In a recent paper, Inderfurth (2004) shows that in contrast to what is stated in literature, the optimal policy in the random yield model can be of a non-linear type for uniformly distributed demand and yield. The analysis provided by the author concerns errors that are such that the standard deviation of the quantity received is proportional to the quantity ordered. This type of errors will be referred to as multiplicative errors in the rest of the paper. Demand and yield are assumed to be uniformly distributed between zero and an upper limit which also restricts the analysis.

Our paper extends the work of Inderfurth (2004) by examining two types of errors:

- Additive errors case: the standard deviation of the received quantity is independent of the quantity ordered;

- Multiplicative errors case: the standard deviation of the quantity received is proportional to the quantity ordered.

In the first case, errors in the received quantity may stem from administrative errors made by the supplier recording for instance a 7 as a 9 in the ordering process. In this case, the variability of errors does not depend on the ordered quantity. In the second case, which is also known as stochastically proportional yield model in the literature, the variability of errors varies with the ordered quantity. Factors such as theft can probably be modeled in this way since the higher is the ordering quantity, the higher will be the variability of the quantity stolen. For a more detailed discussion on the type of errors, the reader is referred to Sahin (2004).

We show that, depending on values that system parameters take, the optimal quantity to order may not be in the form of a Newsvendor type solution adjusted by the average error rate. We then develop a complete analysis that enables to determine the optimal order quantity in presence of errors for all values of system parameters. Secondly, we evaluate the benefit that would stem from eliminating the uncertainty on the quantity received by comparing the optimal costs associated with a model without errors and a model where errors perturb the quantity effectively received from supplier. We then analyze the model for a normally distributed demand and received quantity and strengthen results given in the first part. Table 1 represents our contributions compared with the work of Inderfurth (2004).

The paper is organized as follows : in section 1, we describe the basic error free model (Model 0) and the model with errors (Model 1). In section 2, we derive the optimal order quantity when demand is uniformly distributed for both types of errors. The benefit of making the supplier reliable is analyzed in section 3 . In section 4 , we extend the model for a normally distributed demand. Finally, section 5 concludes the paper.

\section{Model Description}

\subsection{Notations}

The following notations are used: 


\begin{tabular}{lll}
\hline & Inderfurth 2004 & Our Paper \\
\hline $\begin{array}{l}\text { Type of errors } \\
\text { modeled }\end{array}$ & Multiplicative errors & $\begin{array}{l}\text { Additive and Multiplicative } \\
\text { errors }\end{array}$ \\
\hline $\begin{array}{l}\text { Demand and er- } \\
\text { ror distributions }\end{array}$ & $\begin{array}{l}\text { Demand and error are uni- } \\
\text { formly distributed between } \\
0 \text { and an upper bound }\end{array}$ & $\begin{array}{l}\text { 1. Demand and error are } \\
\text { uniformly distributed be- } \\
\text { tween a lower and an upper } \\
\text { bound } \\
\end{array}$ \\
& & $\begin{array}{l}\text { 2. Demand and error are } \\
\text { normally distributed }\end{array}$ \\
\hline $\begin{array}{l}\text { Scope of the anal- } \\
\text { ysis }\end{array}$ & The optimal order policy & $\begin{array}{l}\text { The optimal quantity to or- } \\
\text { der and the associated ex- } \\
\text { pected optimal cost }\end{array}$ \\
\hline
\end{tabular}

Table 1 Main contributions

- $Q_{0}$ : the order quantity in the basic model (Model 0)

- $Q_{0}^{*}$ : the optimal value of $Q_{0}$

- $Q_{1}$ : the order quantity in the Random Yield Model (Model 1)

- $Q_{1}^{*}$ : the optimal value of $Q_{1}$

$-h$ : the unit overage cost

$-u$ : the unit underage cost $*$

$-x$ : the random variable representing demand

$-\mu_{x}$ : the expected demand

- $\sigma_{x}$ : the standard deviation of $x$

- if $x$ is uniform let:

- $U_{x}$ : the upper bound of $x$ which is given by $U_{x}=\mu_{x}+\sqrt{3} \sigma_{x} \geq 0$

$-L_{x}$ : the lower bound of $x$ which is given by $L_{x}=\mu_{x}-\sqrt{3} \sigma_{x} \geq 0$

- $f(F)$ : pdf (cdf) characterizing the demand

$-\phi(\Phi)$ : the standard normal pdf (cdf)

$-Q_{A}$ : the random variable representing the quantity received

- if $Q_{A}$ is uniform let:

- $U_{Q_{A}}$ : the upper bound of $Q_{A}$

$-L_{Q_{A}}$ : the lower bound of $Q_{A}$

- $g\left(Q_{A}\right)$ : pdf characterizing $Q_{A}$

$-\mu_{Q_{A}}$ : the expected quantity received

- $\sigma_{Q_{A}}$ : the standard deviation of $Q_{A}$

* without loss of generality, in the rest of the paper, we set $h=1$ and use $u=k . h$ where $k \in[0.5,10]$

\subsection{Model 0: The basic model without errors}

The expected cost function associated with the basic (standard) on-period Newsvendor model with zero initial inventory is given by: 


$$
C_{0}\left(Q_{0}\right)=k h \int_{Q_{0}}^{+\infty}\left(x-Q_{0}\right) f(x) d x+h \int_{0}^{Q_{0}}\left(Q_{0}-x\right) f(x) d x
$$

Which is minimized for $Q_{0}^{*}$ such that:

$$
F\left(Q_{0}^{*}\right)=\frac{k}{k+1} .
$$

For a uniformly distributed demand, the optimal order quantity would be given by

$Q_{0}^{*}=\mu_{x}+\sqrt{3} \sigma_{D} \frac{k-1}{k+1}$, and the corresponding expected optimal cost is $C_{0}\left(Q_{0}^{*}\right)=\frac{\sqrt{3} k h \sigma_{x}}{k+1}$.

For a normally distributed demand, the optimal order quantity is $Q_{0}^{*}=\mu_{x}+\sigma_{x} \Phi^{-1}\left(\frac{k}{k+1}\right)$ and the optimal expected cost is $C_{0}\left(Q_{0}^{*}\right)=\sigma_{x} h(k+1) \phi\left\{\Phi^{-1}\left[\frac{k}{k+1}\right]\right\}$.

\subsection{Model 1: The model with errors}

\section{Modeling of Errors:}

In a general setting, the received quantity $Q_{A}$ can be modeled by:

$Q_{A}=\gamma Q_{1}+\xi$ where

- $Q_{1}$ is the ordered quantity

$-\gamma$ and $\xi$ are random with respectively $\left(\mu_{\gamma}, \sigma_{\gamma}\right)$ and $\left(\mu_{\xi}, \sigma_{\xi}\right)$ as parameters $\mu_{j}$ being the mean of the random variable $\mathrm{j}$ and $\sigma_{j}$ its standard deviation

One may distinguish two particular cases from this general expression:

- The additive case: the received quantity is given by $Q_{A}=Q_{1}+\xi$, as a result we have $\mu_{Q_{A}}=Q_{1}+\mu_{\xi}$. Without loss of generality we will set $\mu_{\xi}=0$ for the analysis of this model since if $\mu_{\xi} \neq 0$, we can easily show that the optimal order quantity is simply shifted by the constant value $\mu_{\xi}$. In the additive case we therefore have $\mu_{Q_{A}}=Q_{1}$ and $\sigma_{Q_{A}}=\sigma_{\xi}$ which is independent of the ordered quantity.

- The multiplicative case: the received quantity is given by $Q_{A}=\gamma Q_{1}$, as a result we have $\mu_{Q_{A}}=\mu_{\gamma} Q_{1}$ and $\sigma_{Q_{A}}=\sigma_{\gamma} Q_{1}$ which is proportional to the ordered quantity.

Note that the analysis pertaining to the cases $Q_{A}=\mu_{\gamma} Q_{1}+\xi$ and

$Q_{A}=\gamma Q_{1}+\mu_{\xi}$ can be deduced from our analyses on the additive and the multiplicative cases. 


\section{Expected cost function:}

To develop the expected total cost associated with the random yield problem, the following observation must be made: the inventory at the end of the period will be one of the two cases: (a) $x \geq Q_{A}$ and (b) $x \leq Q_{A}$.

The first case triggers an underage situation while the second generates excessive inventory. The cost incurred will be given by:

$$
\text { Cost }=k \cdot h \operatorname{Max}\left(x-Q_{A}, 0\right)+h \operatorname{Max}\left(Q_{A}-x, 0\right)
$$

The expected total cost function of the system is therefore given by:

$$
\begin{array}{r}
C_{1}\left(Q_{1}\right)=k \cdot h \int_{Q_{A}=0}^{\infty} \int_{x=Q_{A}}^{\infty}\left(x-Q_{A}\right) f(x) g\left(Q_{A}\right) d x d Q_{A}+ \\
h \int_{Q_{A}=0}^{\infty} \int_{x=0}^{Q_{A}}\left(Q_{A}-x\right) f(x) g\left(Q_{A}\right) d x d Q_{A}
\end{array}
$$

The following remarks can be made:

- In contrast to Inderfurth (2004), this formulation supposes that the supplier will not be paid for undelivered quantities which seems to be a realistic assumption. Note however that the analysis presented in this paper can easily be modified to consider the case where the supplier is paid for the whole ordered quantity. In the last case, it can be shown that the expected profit function becomes $C_{1}\left(Q_{1}\right)+w\left(Q_{1}-E\left(Q_{A}\right)\right)$ where $w$ is the product unit purchase price.

- Note also that, although we assume that $Q_{A}$ is perfectly known, the cost associated with the inspection process is deliberately not part of our model. Estimates of this cost can be found by various studies and are assumed not to vary with the model parameters. Thus, the expected total cost of the model with errors can be deduced by integrating this cost component.

- Remark that we assume that there is no initial inventory. If there is an initial inventory, the optimal policy may not be of order-up-to type. However, the case with an initial inventory can be handled by following the methodology developed in this paper. In particular, we will show that in the multiplicative errors case, the ordering quantity is not a linear function of the initial inventory level (cf Appendix 4). 


\section{The optimal order decision when demand and errors are uniformly distributed}

\subsection{The additive case}

If demand is uniformly distributed, we have $f(x)=\frac{1}{U_{x}-L_{x}}$ for $L_{x} \leq x \leq U_{x}$ and $f(x)=0$ otherwise

We develop the analysis pertaining to this case in two steps. We first recall results existing in literature. By following a more thorough approach, we show that these results are not valid for all values of system parameters. This pushes us to conduct a more refined analysis enabling to extend results found in the earlier studies.

Previous results from literature:

According to Noori and Keller (1986), whatever the distribution of $Q_{A}$ is, the optimal quantity to order will be given by:

$$
Q_{1}^{*}=Q_{0}^{*}=\mu_{x}+\sqrt{3} \sigma_{x} \frac{k-1}{k+1}
$$

We note there that the optimal order quantity is independent of the standard deviation of the received quantity $\sigma_{\xi}$. This result is somewhat surprising, since an augmentation of $\sigma_{\xi}$ increases the variability of the quantity received which should affect the optimal order quantity.

Extension of the results:

In Noori and Keller (1986), it is stated that the result above holds for all values of system parameters. We show in this section that this may not be true.

We consider the case of a uniformly distributed received quantity where $g\left(Q_{A}\right)=\frac{1}{U_{Q_{A}}-L_{Q_{A}}}$ for $L_{Q_{A}} \leq Q_{A} \leq U_{Q_{A}}$ and $g\left(Q_{A}\right)=0$ otherwise with $U_{Q_{A}}=Q_{1}+\sigma_{\xi} \sqrt{3}$ and $L_{Q_{A}}=Q_{1}-\sigma_{\xi} \sqrt{3}$.

In order to show that the previous result is not verified for all values of system parameters, we consider a deterministic demand which is a particular case of the model above $\left(\mu_{D}=D\right.$ and $\left.\sigma_{D}=0\right)$. We can easily show that the optimal order quantity when demand is deterministic and the received quantity is uncertain is as follows:

$$
Q_{1}^{*}=D+\sqrt{3} \sigma_{\xi} \frac{k-1}{k+1}
$$

We remark that $Q_{1}^{*}$ depends on $\sigma_{\xi}$, which is not compatible with the result given in (5). In fact, (5) is valid only for specific values of the standard deviation $\sigma_{\xi}$ of $Q_{A}$. Indeed, given that $x$ and $Q_{A}$ are bounded, this result is associated with a particular positioning between the distribution of demand and $Q_{A}$ which can be described by $U_{Q_{A}} \leq U_{x}$ and $L_{Q_{A}} \geq L_{x}$, i.e. Configuration 2 in Figure 1. If values of system parameters change, several 
configurations of demand and error distributions should be considered to correctly formulate the expected cost. These configurations are presented in Figure 1.

- Configuration 1: the variability of the received quantity is higher but the distribution of the received quantity is within the distribution of demand: $\operatorname{Max}\left(Q_{A}\right)=U_{Q_{A}} \leq \operatorname{Max}(x)=U_{x}$ and $\operatorname{Min}\left(Q_{A}\right)=L_{Q_{A}} \geq$ $\operatorname{Min}(x)=L_{x}$

- Configuration 2: the variability of the received quantity is such that its distribution exceeds by one side the distribution of demand:

- if $k>1: \operatorname{Max}\left(Q_{A}\right)=U_{Q_{A}} \geq \operatorname{Max}(x)=U_{x}$

- if $k<1: \operatorname{Min}\left(Q_{A}\right)=L_{Q_{A}} \leq \operatorname{Min}(x)=L_{x}$

- if $k=1$ : configuration 2 does not exist

- Configuration 3: the distribution of $Q_{A}$ is no longer in the field of variation of variation of demand: $\operatorname{Max}\left(Q_{A}\right)=U_{Q_{A}} \geq \operatorname{Max}(x)=U_{x}$ and $\operatorname{Min}\left(Q_{A}\right)=L_{Q_{A}} \leq \operatorname{Min}(x)=L_{x}$

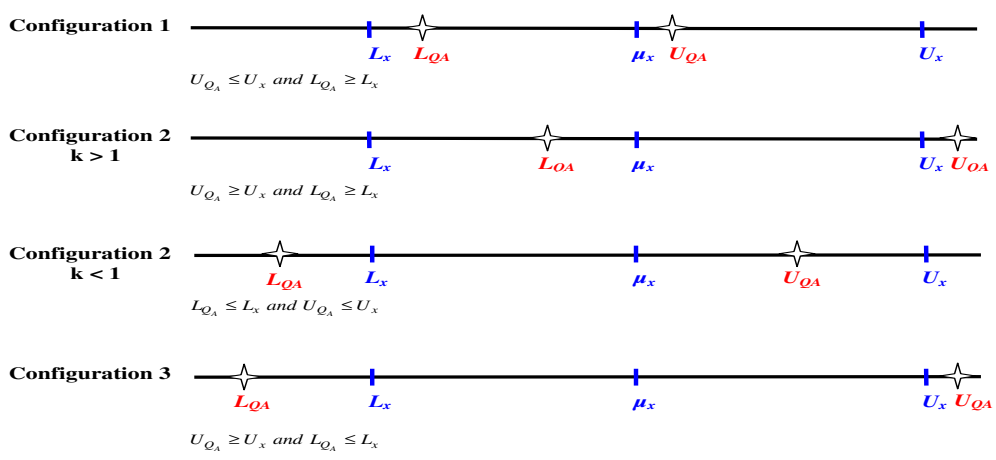

Fig. 1 Positions between distributions of $x$ and $Q_{A}$

In order to express the overall optimal order decision over all possible configurations, we proceed in several steps:

1. We develop the expected total cost pertaining to each configuration

2. We verify the convexity of the total cost function and derive the optimal quantity and cost for each configuration

3. For a given configuration, the expression of the optimal quantity and constraints resulting from the positions of distribution of $x$ and $Q_{A}$ enable to define an interval of $\sigma_{\xi}$ for which results obtained are valid

Note that from a theoretical point of view, in our analysis, we consider all possible values of $\sigma_{\xi}$, including the ones such that $\sigma_{\xi} \geq \sigma_{D}$. This enables us to identify 3 different intervals of variation of $\sigma_{\xi}$, each being associated with one of our configurations. 
3.1.1 Configuration 1 This configuration corresponds to the situation where $U_{Q_{A}} \leq U_{x}$ and $L_{x} \leq L_{Q_{A}}$ :

Result 1 In configuration 1:

For any value of $k$, the optimal order quantity is $Q_{1}^{*}=Q_{0}^{*}=\mu_{x}+\sigma_{x} \sqrt{3} \frac{k-1}{k+1}$ with an optimal cost

$C_{1}\left(Q_{1}^{*}\right)=\frac{h\left(12 k \sigma_{x}^{2}+(k+1)^{2} \sigma_{\xi}^{2}\right)}{4 \sqrt{3}(k+1) \sigma_{x}}$

Proof For technical detail consideration cf Appendix 1.1

In contrast to what is stated in Noori and Keller (1986), the result above is valid only if $Q_{1}^{*}$ verifies $Q_{1}^{*}+\sqrt{3} \sigma_{\xi} \leq U_{x}$ for the case $k \geq 1$ and $Q_{1}^{*}-\sqrt{3} \sigma_{\xi} \geq$ $L_{x}$ for the case $k \leq 1$ (a more detailed discussion on this constraint is developed in Result 4). In Configuration 1, we confirm results obtained in Noori and Keller (1986), and we remark that an increase of $\sigma_{\xi}$ implies additional overage and underage costs but those costs are compensated by each other. So, the optimal ordering quantity does not depend on the standard deviation of the received quantity.

In this configuration, the overage and underage costs are compensated by each other is due to the symmetry of a uniform distribution. We analyzed configuration 2 for a triangular distribution of the received quantity and we showed that the optimal order quantity changes and depends on $\sigma_{\xi}$. So the result given in (5) is not valid for any distribution of the received quantity as stated in Noori and Keller (1986).

3.1.2 Configuration 2 This configuration corresponds to the situation such that $U_{Q_{A}} \geq U_{x}$ and $L_{Q_{A}} \geq L_{x}$ for the case $k \geq 1$ and $U_{Q_{A}} \leq U_{x}$ and $L_{x} \geq L_{Q_{A}}$ for the case $k \leq 1$.

\section{Result 2 In Configuration 2:}

- If $k>1$ :

The optimal order quantity is $Q_{1}^{*}=Q_{0}^{*}+\sqrt{3}\left(\sqrt{\sigma_{\xi}}-\sqrt{\frac{2}{k+1} \sigma_{x}}\right)^{2}$ with an optimal expected cost $C_{1}\left(Q_{1}^{*}\right)=\sqrt{3} h\left(\sigma_{x}+\sigma_{\xi}\right)-4 \frac{h \sqrt{2 \sigma_{x} \sigma_{\xi}}}{\sqrt{3(k+1)}}$

- If $k<1$ :

The optimal order quantity is $Q_{1}^{*}=Q_{0}^{*}-\sqrt{3}\left(\sqrt{\sigma_{\xi}}-\sqrt{\frac{2 k}{k+1} \sigma_{x}}\right)^{2}$ with an optimal expected cost $C_{1}\left(Q_{1}^{*}\right)=\sqrt{3} h k\left(\sigma_{x}+\sigma_{\xi}\right)-4 \frac{h k^{2} \sqrt{2 \sigma_{x} \sigma_{\xi}}}{\sqrt{3 k(k+1)}}$

Proof For technical detail considerations cf Appendix 2.1 
We note there that:

- In contrast to the first configuration, the optimal order quantity depends on the standard deviation of the received quantity $\sigma_{\xi}$ and this is as expected since an increase of the variability of received quantity implies an increase of both underage and overage costs. So the decision to order more or less than the model 0 depends on the value of the parameter $k$ : for $k>1$, the underage penalty is more important than the overage one, we have rather to order more to avoid shortage situation

- The optimal order quantity is increasing (decreasing) in $\sigma_{\xi}$ for $k>1$ $(k<1)$

- Configuration 2 does not exist for the particular case of $k=1$ since the optimal order quantity in Configuration 1 for this case is the expected demand $\mu_{x}$

3.1.3 Configuration 3 This configuration corresponds to the situation such that $L_{Q_{A}} \leq L_{x}$ and $U_{x} \leq U_{Q_{A}}$ for the two cases $(k \leq 1$ and $k \geq 1)$.

Result 3 In configuration 3:

For any value of $k$, the optimal order quantity is $Q_{1}^{*}=\mu_{x}+\sigma_{\xi} \sqrt{3} \frac{k-1}{k+1}$ with an optimal cost

$C_{1}\left(Q_{1}^{*}\right)=\frac{h\left((k+1)^{2} \sigma_{x}^{2}+12 k \sigma_{\xi}^{2}\right)}{4 \sqrt{3}(k+1) \sigma_{\xi}}$

Proof For technical detail considerations cf Appendix 3.1

- As in the previous configuration, the optimal order quantity increases (decreases) with the standard deviation of the received quantity for $k \geq 1$ $(k \leq 1)$

- For this configuration, which corresponds to high values of $\sigma_{\xi}$, the optimal order quantity does not depend on the standard deviation of demand: for a given $\sigma_{\xi}$, an increase of $\sigma_{x}$ will increase both underage and overage costs but those costs will be compensated by each other. Note that formulas of configuration 3 can be deduced from the ones of configuration 1 by exchanging $\sigma_{x}$ and $\sigma_{\xi}$

- Note also that the result we obtained in (6), for deterministic demand which represents a particular case of configuration 3 is compatible with the result we show for this configuration

Result 4 : Summary of results:

Based on expressions of optimal quantities obtained for each configuration and the hypothesis on the positions of distributions of demand and the received quantity, we can deduce the following intervals of variation of $\sigma_{\xi}$ for which previous results hold:

- Case $\boldsymbol{A}: k \geq 1$ : The following figure shows the intervals of values of $\sigma_{\xi}$ in which each configuration is defined: 


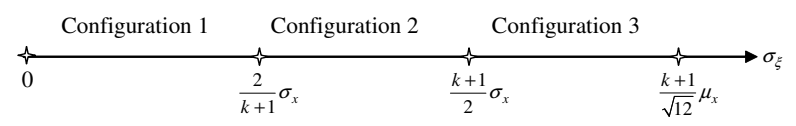

- Case B: $\sqrt{3} \frac{\sigma_{x}}{\mu_{x}} \leq k \leq 1$ : The following figure shows the intervals of values of $\sigma_{\xi}$ in which each configuration is defined:

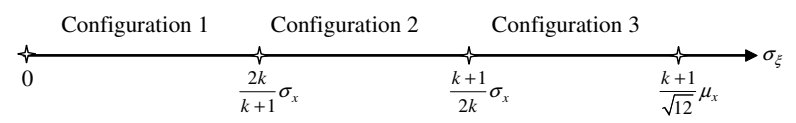

- Case C: $0 \leq k \leq \sqrt{3} \frac{\sigma_{x}}{\mu_{x}}$ : The following figure shows the intervals of values of $\sigma_{\xi}$ in which each configuration is defined:

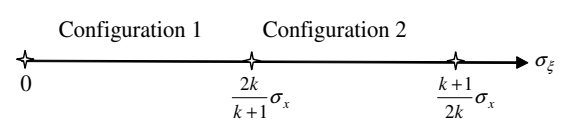

Proof For technical detail considerations cf Appendix 1.2, 2.2 and 3.2.

Note that:

- We have $\frac{2}{k+1} \sigma_{x} \leq \frac{k+1}{2} \sigma_{x} \leq \frac{k+1}{\sqrt{12}} \mu_{x}$ for Case A since $k \geq 1$ and $L_{x} \geq 0$

- We have $\frac{2 k}{k+1} \sigma_{x} \leq \frac{k+1}{2 k} \sigma_{x} \leq \frac{k+1}{\sqrt{12}} \mu_{x}$ for Case B since $\sqrt{3} \frac{\sigma_{x}}{\mu_{x}} \leq k \leq 1$

- For the case $k \leq 1$, an assumption on parameters of demand must be made to assure the existence of Configuration 3: this assumption assures that the lower boundary of $Q_{A}$ reaches zero in Configuration 3 (and not in Configuration 2). So, in the case $k \leq 1, C V_{x}=\frac{\sigma_{x}}{\mu_{x}}$ must satisfy $C V_{x} \leq \frac{k}{\sqrt{3}}$ to assure the existence of Configuration 3. Thus the assumption made on $k\left(\sqrt{3} \frac{\sigma_{x}}{\mu_{x}} \leq k\right)$ for the case $k \leq 1$. For values of $k$ such that $k \leq \sqrt{3} \frac{\sigma_{x}}{\mu_{x}}$ the maximal value that can take $\sigma_{\xi}$ (to assure positive value of lower boundary of $\left.Q_{A}\right)$ is between $\frac{2 k}{k+1} \sigma_{x}$ and $\frac{k+1}{2 k} \sigma_{x}$ (Configuration 2) 
- The continuity of optimal order quantities and costs at each critical value of $\sigma_{\xi}$ is also verified. For example in configuration 2 and for $k \geq 1$, by setting $\sigma_{\xi}=\frac{2}{k+1} \sigma_{x}$, we retrieve the result of the second configuration $Q_{1}^{*}=Q_{0}^{*}$

Figure 2 represents the variation of the optimal order quantity with $\sigma_{\xi}$ for $\mu_{x}=10, \sigma_{x}=3$ :

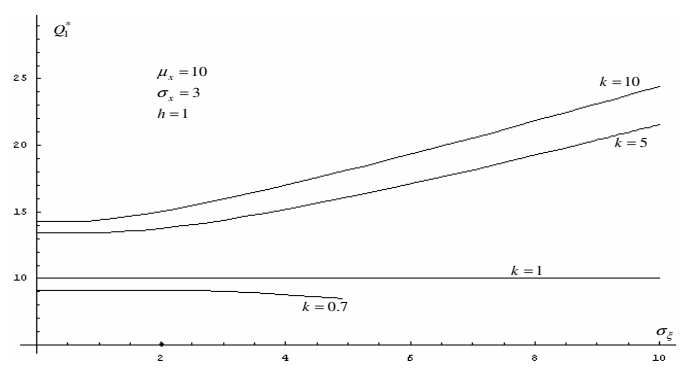

Fig. 2 Variation of $Q_{1}^{*}$ with $\sigma_{\xi}$

Remark: if an initial inventory $I$ is taken into account, we show that the results found in this section are slightly modified and the quantity ordered from supplier is given by $\operatorname{Max}\left(0, Q_{1}^{*}-I\right)$.

\subsection{The multiplicative case}

The received quantity in the multiplicative case is $Q_{A}=\gamma Q_{1}$ with $\mu_{Q_{A}}=\mu_{\gamma} Q_{1}$ and $\sigma_{Q_{A}}=\sigma_{\gamma} Q_{1}$ (cf section 1.3). We have also $g\left(Q_{A}\right)=\frac{1}{\left(U_{\gamma}-L_{\gamma}\right) Q_{1}}$ for $Q_{1} L_{\gamma} \leq Q_{A} \leq Q_{1} U_{\gamma}$ (=0 otherwise) where $U_{\gamma}=\mu_{\gamma}+\sigma_{\gamma} \sqrt{3}$ and $L_{\gamma}=\mu_{\gamma}-\sigma_{\gamma} \sqrt{3}$.

Previous results from literature: For such case, Noori and Keller (1986) show that:

$$
Q_{1}^{*}=\frac{\mu_{\gamma}}{\mu_{\gamma}^{2}+\sigma_{\gamma}^{2}} Q_{0}^{*}
$$

This result is also found by Gerchak, Parlar and Vickson (1986) (with the assumption that the upper bound of demand is less than the yield one and by setting the initial stock equal to zero). It is also provided in Ehrhardt and Taube (1987) but also under the same condition mentioned above.

Extension of the results:

In order to show that the result in (7) is not valid for all values of model 
parameters, we analyze the model for a deterministic demand $\left(\mu_{D}=D\right.$ and $\left.\sigma_{D}=0\right)$. We can easily show that the optimal order quantity when received quantity is uncertain is given by:

$$
Q_{1}^{*}=\frac{\sqrt{(k+1) D^{2}}}{\sqrt{-2 \sqrt{3}(k-1) \mu_{\gamma} \sigma_{\gamma}+(k+1)\left(\mu_{\gamma}^{2}+3 \sigma_{\gamma}^{2}\right)}}
$$

This result is clearly not compatible with (7) which is only valid for specific values of $\sigma_{\gamma}$. Again, by using the same logic as the additive case (cf Page 8 ), we have 3 configurations depending on positions of the distributions of demand and errors, and we show that (7) corresponds to the case where the distribution of $Q_{A}$ is included in demand's one.

Because of the complexity of formulas that express the optimal cost, those are not provided in this paper but can be found in Rekik (2003). As in the additive case, based on expressions of optimal quantities and the hypothesis concerning the positions of distributions of $x$ and $Q_{A}$, we deduce the interval of $\sigma_{\gamma}$ for which each result is valid (we consider $\sigma_{i j}$ as the critical value of $\sigma_{\gamma}$ which permits the transit from Configuration $i$ to Configuration $j$ ).

Theorem 1 The optimal order decision pertaining to a situation where $\gamma$ has $\left(\mu_{\gamma}, \sigma_{\gamma}\right)$ as parameters can be obtained by determining the optimal order decision associated with the normalized case where the parameters of the distribution of $\gamma$ are given by $\left(1, \frac{\sigma_{\gamma}}{\mu_{\gamma}}\right)$. The optimal order quantity of the first case is equal the optimal order quantity of the second one divided by $\mu_{\gamma}$.

Proof Consider a first model with error parameter setting $\left(\mu_{\gamma}, \sigma_{\gamma}\right)$ and an order quantity equal to $Q 1$. Consider also the second model with error parameter setting $\left(1, \frac{\sigma_{\gamma}}{\mu_{\gamma}}\right)$ and an order quantity equal to $Q 2$. If we assume that $Q 2=\mu_{\gamma} Q 1$ then the two models are equivalent since the distribution of the received quantity is the same in both models.

In order to compare our results with those that exist in literature, we assume that $\mu_{\gamma} \neq 1$ in the formulation of our model. We use the same method as the additive case: $i$ ) determination of the total cost function; $i i$ ) verification of convexity and deduction of the optimal quantity and cost; iii) determination of the interval of $\sigma_{\gamma}$ where the result is valid $i v$ ) verification of the expressions and the sequence of critical values of $\sigma_{\gamma}$.

Result 5 Expressions of the optimal order quantity for each configuration are as follows: 


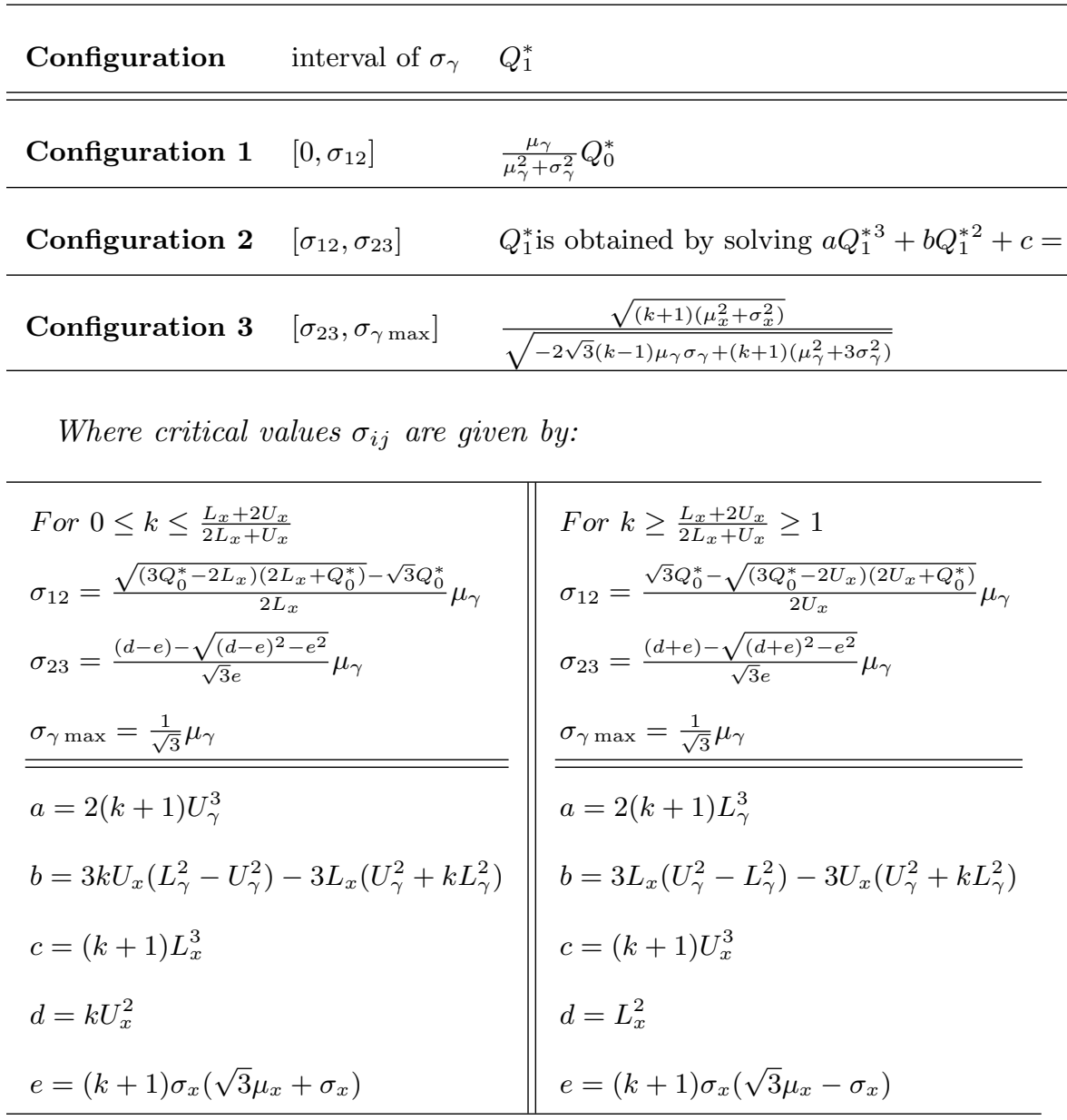

Proof The logic used is the same as the additive case, technical detail considerations are not presented in this paper but they can be found in Rekik (2003)

Note that for the case $k \leq \frac{L_{x}+2 U_{x}}{2 L_{x}+U_{x}}$ and again like the additive case, an assumption on $k, \sigma_{x}$ and $\mu_{x}$ must be made to assure the existence of Configuration 3 and to assure that the lower boundary of $Q_{A}$ reaches zero in Configuration 3 (and not in Configuration 2). So $C V_{x}=\frac{\sigma_{x}}{\mu_{x}}$ must satisfy $C V_{x} \leq \frac{\sqrt{3}-\sqrt{(3-k)(k+1)}}{k-2}$ to assure the existence of Configuration 3, otherwise the maximum value that can take $\sigma_{\gamma}$ is between $\sigma_{23}$ and $\sigma_{34}$.

We notice here that all results in the literature except the one of Inderfurth (2004) consider only Configuration 1. The analysis of Inderfurth (2004) is a particular case of our analysis with the assumption that $L_{x}=L_{\gamma}=0$. 
With such an assumption, Configuration 3 does not exist and Configuration 2 is only valid for the case $k \geq 2$. For such case we can easily verify the result provided by the author $Q_{1}^{*}=\frac{U_{x}}{U_{\gamma}} \sqrt{\frac{k+1}{3}}$.

Below (see Figure 3) we present the variation of the optimal order quantity with $\sigma_{\gamma}$ for a demand with parameters $\mu_{x}=10$ and $\sigma_{x}=3$ and for $\mu_{\gamma}=1$ :

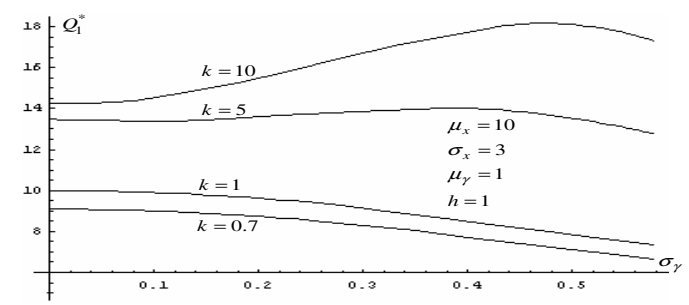

Fig. 3 Variation of $Q_{1}^{*}$ with $\sigma_{\gamma}$ for different $k$

Note that:

- As the additive case, the effect of $k$ on the optimal order quantity is as expected intuitively

- The optimal order quantity is inversely proportional to $\mu_{\gamma}$ and this is intuitively expected: if the supplier delivers less than the company orders, it must order a larger amount

- In order to avoid negative value of received quantity, $\mu_{\gamma}$ and $\sigma_{\gamma}$ must satisfy $\mu_{\gamma}-\sqrt{3} \sigma_{\gamma} \geq 0$, this is why $\sigma_{\gamma \max }=\frac{\mu_{\gamma}}{\sqrt{3}}$

- In some situations where $\mathrm{k}$ is small such as the case $k=0.7$ presented in Figure 3, Configuration 3 does not exist because the lower boundary of $Q_{A}$ reaches zero in Configuration 2

- The effect of $\sigma_{\gamma}$ : like the additive case, the variation of optimal order quantity with $\sigma_{\gamma}$, depends on value taken by the parameter $k$ :

(a) If $k>\frac{L_{x}+2 U_{x}}{2 L_{x}+U_{x}}$ : underage situation is more penalizing in term of costs: an increase of $\sigma_{\gamma}$ will increase the probability of falling in the underage case, so increasing the order quantity will help the decrease of this probability. But from a certain value of $\sigma_{\gamma}$, the increase of the optimal order quantity will generate more important costs stemming from the increase of the variability of the received quantity since its boundaries are proportional to the order quantity, then, from this value of $\sigma_{\gamma}$, we have rather to order less to diminish the variability of $Q_{A}$, so $Q_{1}^{*}$ decreases. As a consequence, in the case $k>\frac{L_{x}+2 U_{x}}{2 L_{x}+U_{x}}$, we have two phenomena linked to the increase of $\sigma_{\gamma}$ which interfere: i) if $\sigma_{\gamma}$ increases, the probability to fall in a underage situation gets higher, increasing order quantity will decrease the probability of such 
situation. ii) If $\sigma_{\gamma}$ increases, the field of variation of received quantity is higher and so its variability which is equal to $\sigma_{\gamma} Q_{1}$ is bigger and as a consequence total cost function gets bigger. Decreasing order quantity is a way to reduce costs since the boundaries of the distribution of $Q_{A}$ depends on the order quantity $Q_{1}$.

(b) If $k<\frac{L_{x}+2 U_{x}}{2 L_{x}+U_{x}}$ : overage costs are less important than underage ones. An increase of $\sigma_{\gamma}$ will increase both underage and overage costs, so decreasing the order quantity will be the best solution to decrease the total expected cost function by decreasing the variability of the received quantity.

Remark: if an initial inventory $I$ is taken into account, we observe that the ordering quantity is not a linear function of $I$ (cf Appendix 4).

\section{The benefit of making the supplier reliable}

It would be worthwhile to know the benefit of making supplier $100 \%$ reliable as a function of system parameters. This is achieved by using the ratio:

$$
R=\frac{C_{1}\left(Q_{1}^{*}\right)-C_{0}\left(Q_{0}^{*}\right)}{C_{1}\left(Q_{1}^{*}\right)}
$$

\subsection{The additive case}

We consider in this section, the benefit of making the supplier reliable in the additive case.

Result 6 Expressions of $R$ for the different intervals of variation of $\sigma_{\xi}$ will be given by:

\begin{tabular}{|l|l|l|l|l|}
\hline \multirow{2}{*}{ Conf. } & \multicolumn{2}{|c|}{ Interval of $\sigma_{\xi}$} & \multicolumn{2}{|}{$\mathrm{R}$} \\
\cline { 2 - 3 } Conf. 1 & $k \leq 1$ & {$\left[0, \frac{2 k}{k+1} \sigma_{x}\right]$} & {$\left[0, \frac{2}{k+1} \sigma_{x}\right]$} & $1-\frac{12 k \sigma_{x}^{2}}{12 k \sigma_{x}^{2}+(k+1)^{2} \sigma_{\xi}^{2}}$ \\
\hline Conf. 2 & {$\left[\frac{2 k}{k+1} \sigma_{x}, \frac{k+1}{2 k} \sigma_{x}\right]$} & {$\left[\frac{2}{k+1} \sigma_{x}, \frac{k+1}{2} \sigma_{x}\right]$} & $k \leq 1$ & $1-\frac{3 \sigma_{x}}{3(k+1)\left(\sigma_{x}+\sigma_{\xi}\right)-4 \sqrt{2 k(k+1) \sigma_{x} \sigma_{\xi}}}$ \\
& & $k \geq 1$ & $1-\frac{3 \sigma_{x}}{3(k+1)\left(\sigma_{x}+\sigma_{\xi}\right)-4 \sqrt{2(k+1) \sigma_{x} \sigma_{\xi}}}$ \\
\hline Conf. 3 & {$\left[\frac{k+1}{2 k} \sigma_{x}, \frac{k+1}{\sqrt{12}} \mu_{x}\right]$} & {$\left[\frac{k+1}{2} \sigma_{x}, \frac{k+1}{\sqrt{12}} \mu_{x}\right]$} & $1-\frac{12 k \sigma_{x} \sigma_{\xi}}{(1+k)^{2} \sigma_{x}^{2}+12 k \sigma_{\xi}^{2}}$ \\
\hline
\end{tabular}

For our numerical example $\left(k=5, \mu_{x}=10, \sigma_{x}=3\right.$ and $\left.\sigma_{Q_{A}}=\sigma_{\xi}=4\right)$ we have to order $Q_{1}^{*}=15.19$ with an optimal $\operatorname{cost} C_{1}\left(Q_{1}^{*}\right)=7.50$. If the supplier were $100 \%$ reliable, the optimal order quantity would be the Newsvendor solution $Q_{0}^{*}=13.46$ and the associated cost $C_{0}\left(Q_{0}^{*}\right)=4.33$. So, the benefit 
we get if the supplier is $100 \%$ reliable represents $42 \%$ of the cost of the situation with unreliable supplier.

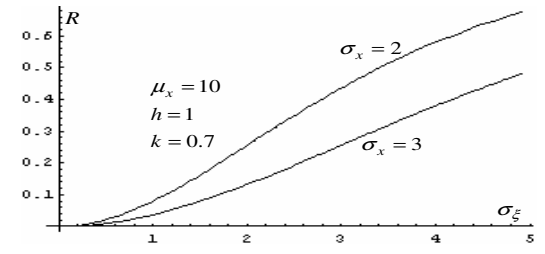

Fig. 4 Variation of $R$ with $\sigma_{\xi}$ with $\sigma_{x}$ for $k=0.7$

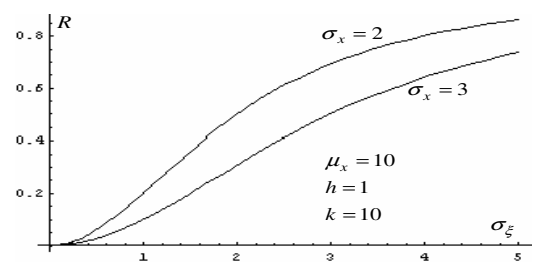

Fig. 5 Variation of $R$ with $\sigma_{\xi}$ with $\sigma_{x}$ for $k=10$

Figure 4 and 5 present the variation of $R$ with $\sigma_{\xi}$ for 2 different values of $\sigma_{x}$ for $k=0.7$ and $k=10$ respectively. As expected $R$ is increasing with $\sigma_{\xi}$, the higher the error made by supplier is, the more important the benefits of making it $100 \%$ reliable is. Note also, by comparing the two figures, that the benefits we get by making the supplier reliable is increasing with $k$ and this is also expected: if $k$ is high, the trade-off between underage and overage costs is more sensitive to supplier's errors. As we can remark, the benefits we make by making our supplier reliable is less important when the variability of demand is more important.

\subsection{The multiplicative case}

We consider in this section, the benefit of making the supplier reliable in the multiplicative case by analyzing the ratio $R$. Again, as in the additive errors case, the ratio $R$ is defined in each configuration and the continuity is checked for each critical level of $\sigma_{\gamma}$. Because of complexity, expressions of $R$ are not provided but they can be found in Rekik (2003). We analyze graphically the variation of $R$ with model's parameters.

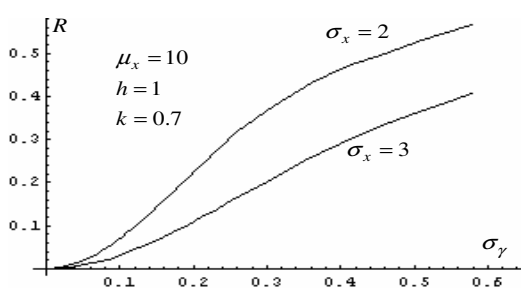

Fig. 6 Variation of $R$ with $\sigma_{\gamma}$ with $\sigma_{x}$ for $k=0.7$

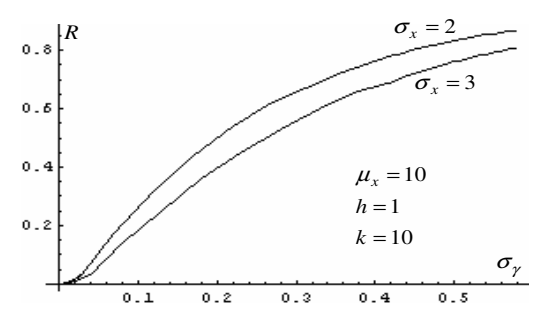

Fig. 7 Variation of $R$ with $\sigma_{\gamma}$ with $\sigma_{x}$ for $k=10$ 
Figure 6 and 7 present the variation of $R$ with $\sigma_{\gamma}$ with $\sigma_{x}$ for $k=0.7$ and $k=10$ respectively. As in the additive case, $R$ is increasing with $\sigma_{\gamma}$ : the higher the error made by supplier is, the more important the benefit of making it $100 \%$ reliable is. Note also that $R$ decreases with $\sigma_{x}$.

\section{The optimal order decision when demand and errors are normally distributed}

In this section, we consider the case of a normally distributed $x$ and $Q_{A}$. Our results confirm the findings we obtained in section 3 for the optimal order quantity and section 4 for the benefits we can get by making the supplier $100 \%$ reliable. Again, we consider the additive and the multiplicative cases.

\subsection{The additive case}

For the additive error case, a closed form analytical solution for the optimal order quantity can be determined under normally distributed demand and errors. In fact, Equation 3 for additive errors will be given by:

$$
\text { Cost }=k \cdot h \operatorname{Max}\left(x-\left(Q_{1}+\xi\right), 0\right)+h \operatorname{Max}\left(\left(Q_{1}+\xi\right)-x, 0\right)
$$

which can be rewritten as:

$$
\text { Cost }=k \cdot h \operatorname{Max}\left((x-\xi)-Q_{1}, 0\right)+h \operatorname{Max}\left(Q_{1}-(x-\xi), 0\right)
$$

We can define an equivalent aggregated demand $x_{e q}=x-\xi$ which is normally distributed with parameters $\mu_{x e q}=\mu_{x}$ and $\sigma_{x e q}=\sqrt{\sigma_{x}^{2}+\sigma_{\xi}^{2}}$. It then appears that the original Newsvendor model with additive errors is simply equivalent to a classical Newsvendor with this equivalent aggregated demand. As a result, the optimal order quantity is given by:

$$
Q_{1}^{*}=\mu_{x e q}+\sigma_{x e q} \Phi^{-1}\left(\frac{k}{k+1}\right)
$$

And the optimal expected cost is:

$$
C_{1}\left(Q_{1}^{*}\right)=\sigma_{x e q} h(k+1) \phi\left\{\Phi^{-1}\left[\frac{k}{k+1}\right]\right\}
$$

Based on the above expression, we obtain the following numerical results for varying values of system parameters (with $\mu_{x}=10$ and $\sigma_{x}=3$ ). 


\begin{tabular}{ccccc}
\hline & $\mathbf{k}=\mathbf{0 . 7}$ & $\mathbf{k}=\mathbf{1}$ & $\mathbf{k}=\mathbf{5}$ & $\mathbf{k}=\mathbf{1 0}$ \\
$\sigma_{\xi}$ & $Q_{1}^{*}$ & $Q_{1}^{*}$ & $Q_{1}^{*}$ & $Q_{1}^{*}$ \\
\hline \hline 0.00 & 9.33 & 10.00 & 12.90 & 14.01 \\
0.50 & 9.32 & 10.00 & 12.94 & 14.06 \\
1.00 & 9.30 & 10.00 & 13.06 & 14.22 \\
1.50 & 9.25 & 10.00 & 13.25 & 14.48 \\
2.00 & 9.20 & 10.00 & 13.49 & 14.81 \\
2.50 & 9.13 & 10.00 & 13.78 & 15.21 \\
3.00 & 9.06 & 10.00 & 14.11 & 15.67 \\
3.50 & 8.97 & 10.00 & 14.46 & 16.16 \\
4.00 & 8.89 & 10.00 & 14.84 & 16.68 \\
\hline
\end{tabular}

Table 2 The optimal order quantity with normal distributions of demand and received quantity - additive case

Note that results we obtained in the case with uniform distributions of $x$ and $Q_{A}$ are still valid:

- $Q_{1}^{*}$ increases with $k$

- An increase (decrease) of $\sigma_{\xi}$ produces an increase (decrease) of $Q_{1}^{*}$ for $k \leq 1(k \geq 1)$ (cf Figure 8)

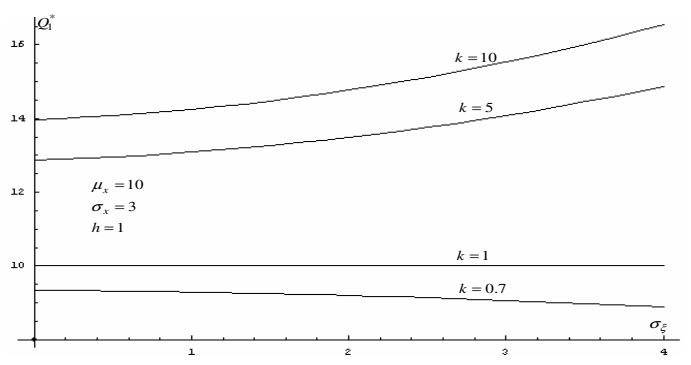

Fig. 8 Variation of $Q_{1}^{*}$ with $\sigma_{\xi}$ for different $k$

As in the case of uniformly distributed demand and received quantity, we analyze the benefits we can get by making the supplier $100 \%$ reliable by studying the ratio $R$ we defined in (9). Variation of $R$ with $\sigma_{\xi}$ is presented in Figure (9) for $k=10$ and $k=0.7$. Figure (10) presents the variation of $R$ with $\sigma_{\xi}$ with $\sigma_{x}$ for $k=3$. Note that we have the same variations as the case with uniform distributions of demand and received quantity. 


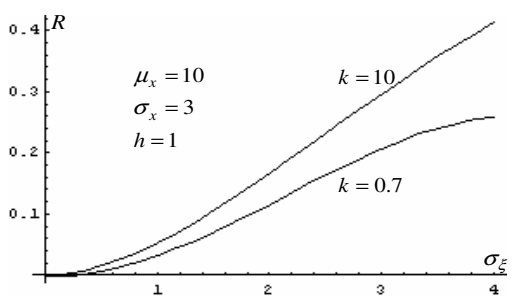

Fig. 9 Variation of $R$ with $\sigma_{\xi}$ for different $k$

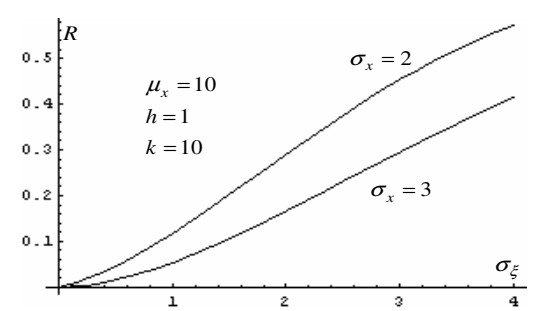

Fig. 10 Variation of $R$ with $\sigma_{\xi}$ with $\sigma_{x}$ for $k=10$

Remark: The equivalence between the original model and the aggregated demand model actually holds for generally distributed demand and additive errors as already noticed in Kök and Shang (2004). In particular, when demand and (additive) errors are uniformly distributed, one can also proceed to the aggregation of distributions to obtain an equivalent demand that follows a triangular distribution. We deliberately did not follow this approach and considered the individual distributions of demand and errors in Section 3.1 in order to develop a generic methodology that is valid for both additive and multiplicative errors. Note however that following the equivalent demand approach would led to a totally similar analysis as the one presented in Section 3.1.

\subsection{The multiplicative case}

A numerical study is performed to optimize the model. Table (7) illustrates the impact of the different parameters on the optimal order quantity for $\mu_{x}=10$ and $\sigma_{x}=3$ :

\begin{tabular}{ccccc}
\hline \hline & $\mathbf{k}=\mathbf{0 . 7}$ & $\mathbf{k}=\mathbf{1}$ & $\mathbf{k}=\mathbf{5}$ & $\mathbf{k}=\mathbf{1 0}$ \\
$\sigma_{\gamma}$ & $Q_{1}^{*}$ & $Q_{1}^{*}$ & $Q_{1}^{*}$ & $Q_{1}^{*}$ \\
\hline \hline 0.00 & 9.33 & 10.00 & 12.90 & 14.01 \\
0.05 & 9.30 & 9.98 & 12.94 & 14.08 \\
0.09 & 9.23 & 9.92 & 13.01 & 14.24 \\
0.13 & 9.13 & 9.84 & 13.11 & 14.47 \\
0.17 & 8.99 & 9.72 & 13.23 & 14.76 \\
0.21 & 8.83 & 9.58 & 13.34 & 15.09 \\
0.25 & 8.65 & 9.42 & 13.45 & 15.43 \\
0.29 & 8.45 & 9.23 & 13.52 & 15.75 \\
0.33 & 8.24 & 9.04 & 13.56 & 16.03 \\
0.37 & 8.02 & 8.82 & 13.55 & 16.25 \\
\hline
\end{tabular}

Table 3 The optimal order quantity for normal distributions of demand and received quantity - multiplicative case $\left(\mu_{\gamma}=1\right)$ 
Again, all the results we obtained in Sections 2.2 and 3.2 are valid:

- The optimal order quantity increases with $k$

- For $k \leq 1$, an increase of $\sigma_{\gamma}$ produces an increase of $Q_{1}^{*}$ (cf Figure 11)

- For $k>1$, as in the case of uniform distributions, there are two phenomena which interfere: for small values of $\sigma_{\gamma}$ the first phenomenon pushes to increase the order quantity. If $\sigma_{\gamma}$ gets higher, the second phenomenon is prevailing and pushes to decrease the field of variation of the received quantity $Q_{A}$ by decreasing the order quantity since the variability of $Q_{A}$ is proportional to this quantity (cf Figure 11)

- The variation of $R$ with $\sigma_{\gamma}, k$ and $\sigma_{x}$ is also expected and is similar to the case with uniform distributions of demand and received quantity (cf Figures 12 and 13).

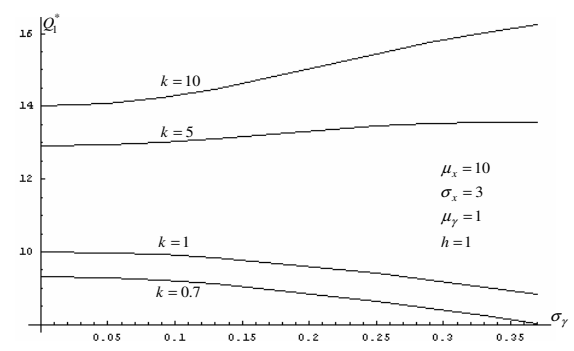

Fig. 11 Variation of $Q_{1}^{*}$ with $\sigma_{\gamma}$ for different $k$

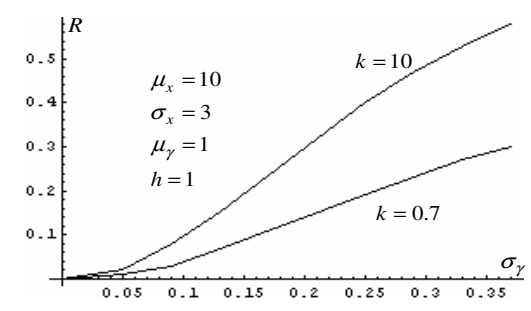

Fig. 12 Variation of $R$ with $\sigma_{\gamma}$ for different $k$

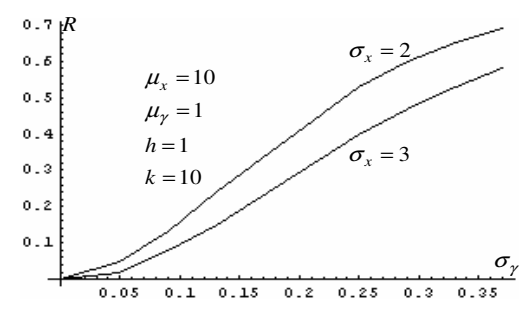

Fig. 13 Variation of $R$ with $\sigma_{\gamma}$ with $\sigma_{x}$ for $k=10$

\section{Conclusion}

In this study, we consider a single stage inventory system with random yield stemming from supplier delivering incorrect ordering quantities. When demand and yield distributions are assumed to be uniform, we show that the earlier results existing in literature are not complete. In other words, we found that previous investigations developed in this area are valid for only a certain range of system parameters. This result motivates us to extend earlier found results and to propose an exhaustive inventory policy. For this 
purpose, we identify the different cases (or configurations) to consider, depending on values that parameters take. We express each of these cases as being an interval of variation of the standard deviation of the received quantity and determine the optimal order decision pertaining to each individual interval. The overall policy (for all possible values of system parameters) is then obtained by juxtaposing the individual optimal policies. This enables us to evaluate the penalty that would stem from using the optimal cost function that is provided in the literature, in comparison with the true optimal cost developed in our analysis.

Our results also show that the random yield issue can lead to significant losses for companies, especially when parameters pertaining to errors are important. In order to quantify this loss, we compare the optimal cost associated with the model without errors to the optimal cost associated with the model with errors. Furthermore, we strengthen our results by analyzing the case of normally distributed demand and errors.

\section{Acknowledgements}

The authors wish to thank two anonymous referees whose comments led to an improved paper in content and presentation

\section{References}

Ehrhardt R. and Taube L. (1987) An inventory model with random replenishment quantities, International Journal of Production Research 25(12), 1795-1804

Gerchak Y., Vickson R. G. and Parlar M. (1988) Periodic review production models with variable yield and uncertain demand, IIE Transactions 20(2), $144-150$

Henig M. and Gerchak Y. (1990) The structure of periodic review policies in the presence of variable yield, Operations Research 38, 634-643

Inderfurth K. (2004) Analytical Solution for a Single-period Productioninventory Problem with Uniformly Distributed Yield and Demand, Central European Journal of Operations Research, 117 - 127

Khouja M. (1990) The Single-Period (Newsvendor) Problem: Literature Review and Suggestions for Future Research, Omega, 537-553

Kök, A., G. and Shang, K., H. (2004) Replenishment and Inspection Policies for Systems with Inventory Record Inaccuracy, Working paper, Fuqua School of Business, Duke University

Lau H.S. (1997) Simple formulas for the expected costs in the news-boy problem: an educational note, European Journal of Operational Research, 100, $557-561$

Lee H.L., Yano C.A. (1988) Production control in multistage systems with variable yield losses, Operations Research 36 (2) 269-278

Noori A.H. and Keller G. (1986) One-period order quantity strategy with uncertain match between the amount received and quantity requisitioned, INFOR 24 (1), 1-11

Rekik Y. (2003) Impacts of the Radio Frequency Identification technology upon the supply chain: approach with the Newsvendor model, MS. Thesis, Ecole 
Centrale Paris, France

Sahin E. (2004) A qualitative and quantitative analysis of the impact of Auto ID technology on the performance of supply chains, PhD Dissertation, Ecole Centrale Paris, France

Silver E.A. (1976) Establishing the order quantity when the amount received is uncertain, INFOR 14 (1), 32-39

Silver E.A., Pyke D.F. and Peterson R. (1998) Inventory Management and Production Planning and Scheduling, Wiley, Chapter 5

Shih W. (1980) Optimal inventory policies when stockouts result from defective products, International Journal of Production Research, 18, 677-685

Yano C.A., Lee H.L. (1995) Lot sizing with random yields: A review, Operations Research 43 (2) 311-334 


\section{APPENDIX}

Appendix 1: Technical details for configuration 1

Appendix 1.1:

In configuration 1 we have three cost functions depending on the value of the received quantity compared with demand's one. We have:

$$
\begin{aligned}
C_{1}^{1}\left(Q_{1}\right)=h \int_{Q_{A}=L_{Q A}}^{U_{Q A}} \int_{x=L_{x}}^{L_{Q A}}\left(Q_{A}-x\right) f(x) g\left(Q_{A}\right) d x d Q_{A} \\
C_{1}^{2}\left(Q_{1}\right)=k . h \int_{Q_{A}=L_{Q A}}^{U_{Q A}} \int_{x=U_{Q A}}^{U_{x}}\left(x-Q_{A}\right) f(x) g\left(Q_{A}\right) d x d Q_{A} \\
C_{1}^{3}\left(Q_{1}\right)=k \cdot h \int_{Q_{A}=L_{Q A}}^{U_{Q A}} \int_{x=Q_{A}}^{U_{Q A}}\left(x-Q_{A}\right) f(x) g\left(Q_{A}\right) d x d Q_{A} \\
+h \int_{Q_{A}=L_{Q A}}^{U_{Q A}} \int_{x=L_{Q_{A}}}^{Q_{A}}\left(Q_{A}-x\right) f(x) g\left(Q_{A}\right) d x d Q_{A}
\end{aligned}
$$

The total cost function is written as the following:

$$
\begin{aligned}
C_{1}\left(Q_{1}\right) & =C_{1}^{1}\left(Q_{1}\right)+C_{1}^{2}\left(Q_{1}\right)+C_{1}^{3}\left(Q_{1}\right) \\
& =\frac{\left.h\left(-6(k-1)\left(Q_{1}-\mu_{x}\right) \sigma_{x}+\sqrt{3}\left(\left(-Q_{1}+\mu_{x}\right)^{2}+3 \sigma_{x}^{2}+\sigma_{\xi}^{2}\right)\right)\right)}{12 \sigma_{x}^{2}}
\end{aligned}
$$

The convexity of $C_{1}\left(Q_{1}\right)$ is clear. Setting $\frac{\partial C_{1}\left(Q_{1}\right)}{\partial Q_{1}}=0$ and solving, we get $Q_{1}^{*}=Q_{0}^{*}=\mu_{x}+\sigma_{x} \sqrt{3} \frac{k-1}{k+1}$ with an optimal cost function equal to: $C_{1}\left(Q_{1}^{*}\right)=\frac{h\left(12 k \sigma_{x}^{2}+(k+1)^{2} \sigma_{\xi}^{2}\right)}{4 \sqrt{3}(k+1) \sigma_{x}}$.

\section{Appendix 1.2:}

The result presented above is valid till:

1. $U_{Q_{A}} \leq U_{x}$ for $k \geq 1$, so $Q_{1}+\sqrt{3} \sigma_{\xi} \leq \mu_{x}+\sqrt{3} \sigma_{x}$.

Replacing $Q_{1}$ by $Q_{1}^{*}=\mu_{x}+\sigma_{x} \sqrt{3} \frac{k-1}{k+1}$ and solving the last inequality, we get $\sigma_{\xi} \leq \frac{2}{k+1} \sigma_{x}$. So, configuration 1 is defined for $\sigma_{\xi} \in\left[0, \frac{2}{k+1} \sigma_{x}\right]$ if $k \geq 1$

2. $L_{x} \leq L_{Q_{A}}$ for $k \leq 1$, so $\mu_{x}-\sqrt{3} \sigma_{x} \leq Q_{1}-\sqrt{3} \sigma_{\xi}$.

Again replacing $Q_{1}$ by $Q_{1}^{*}$ we get $\sigma_{\xi} \leq \frac{2 k}{k+1} \sigma_{x}$. So, configuration 1 is defined for $\sigma_{\xi} \in\left[0, \frac{2 k}{k+1} \sigma_{x}\right]$ if $k \leq 1$ 


\section{Appendix 2: Technical details for configuration 2} Appendix 2.1:

For $k \geq 1$ we have:

$$
\begin{array}{r}
C_{1}^{1}\left(Q_{1}\right)=h \int_{Q_{A}=U_{x}}^{U_{Q_{A}}} \int_{x=L_{x}}^{U_{x}}\left(Q_{A}-x\right) f(x) g\left(Q_{A}\right) d x d Q_{A} \\
C_{1}^{2}\left(Q_{1}\right)=h \int_{Q_{A}=L_{Q_{A}}}^{U_{x=L_{X}}^{U_{X}}}\left(Q_{A}-x\right) f(x) g\left(Q_{A}\right) d x d Q_{A} \\
+k . h \int_{Q_{A}=L_{Q A}}^{Q_{A}} \int_{x=Q_{A}}^{U_{X}}\left(x-Q_{A}\right) f(x) g\left(Q_{A}\right) d x d Q_{A}
\end{array}
$$

The total cost function is written as the following:

$$
\begin{aligned}
& C_{1}\left(Q_{1}\right)=C_{1}^{1}\left(Q_{1}\right)+C_{1}^{2}\left(Q_{1}\right)=\frac{1}{72 \sigma_{x} \sigma_{\xi}}\left(h \left(-(k+1) Q_{1}^{3} C_{\lambda}^{3}+(k+1) \mu_{x}^{2}+\right.\right. \\
& 3 \sqrt{3}(k+1)\left(\sigma_{x} \sigma_{\xi}\right)^{2}+9 \mu_{x}\left((k+1) \sigma_{x}^{2}+2(k-3) \sigma_{x} \sigma_{\xi}+(k+1) \sigma_{\xi}^{2}\right)+ \\
& 3(k+1) Q_{1}^{2}\left(\mu_{x}+\sqrt{3}\left(\sigma_{x}+\sigma_{\xi}\right)\right)-3 Q_{1}\left((k+1) \mu_{x}^{2}+3(k+1) \sigma_{x}^{2}+\right. \\
& \left.\left.\left.6(k-3) \sigma_{x} \sigma_{\xi}+3(k+1) \sigma_{\xi}^{2}+2 \sqrt{3} \mu_{x}(k+1)\left(\sigma_{x}+\sigma_{\xi}\right)\right)\right)\right)
\end{aligned}
$$

The second derivation of $C_{1}\left(Q_{1}\right)$ is equal to $\frac{h(k+1)\left[U_{x}-L_{Q_{A}}\right]}{12 \sigma_{x} \sigma_{\xi}}$ which is all the time positive since $L_{Q A}<U_{x}$, so the convexity of $C_{1}\left(Q_{1}\right)$.

Setting $\frac{\partial C_{1}\left(Q_{1}\right)}{\partial Q_{1}}=0$ and solving we get $Q_{1}^{*}=Q_{0}^{*}+\sqrt{3}\left(\sqrt{\sigma_{\xi}}-\sqrt{\frac{2}{k+1} \sigma_{x}}\right)^{2}$ where: $Q_{0}^{*}=\mu_{x}+\sigma_{x} \sqrt{3}$

By doing the same for the case $k \leq 1$ we get:

$Q_{1}^{*}=Q_{0}^{*}-\sqrt{3}\left(\sqrt{\sigma_{\xi}}-\sqrt{\frac{2 k}{k+1} \sigma_{x}}\right)^{2}$ with an optimal cost function $C_{1}\left(Q_{1}^{*}\right)=$ $\sqrt{3} h k\left(\sigma_{x}+\sigma_{\xi}\right)-4 \frac{\sqrt{2 \sigma_{x} \sigma_{\xi}} h k^{2}}{\sqrt{3 k(k+1)}}$

\section{Appendix 2.2:}

The result obtained above is valid till:

1. If $k \geq 1: L_{Q A} \geq L_{x}$ and $U_{Q A} \geq U_{x}$ so $Q_{1}-\sqrt{3} \sigma_{\xi} \geq \mu_{x}-\sqrt{3} \sigma_{x}$ and $Q_{1}+\sqrt{3} \sigma_{\xi} \geq \mu_{x}+\sqrt{3} \sigma_{x}$.

Again replacing $Q_{1}$ by $Q_{1}^{*}$ we get $\frac{2}{k+1} \sigma_{x} \leq \sigma_{\xi} \leq \frac{k+1}{2} \sigma_{x}$ and this inequality is verified since $k \geq 1$

2. If $k \leq 1$ : by doing the same as the previous case we have $\frac{2 k}{k+1} \sigma_{x} \leq \sigma_{\xi} \leq$ $\frac{k+1}{2 k} \sigma_{D}$. Again the inequality $\frac{2 k}{k+1} \sigma_{x} \leq \frac{k+1}{2 k} \sigma_{D}$ is well verified since $k \leq 1$ 
Appendix 3: Technical details for configuration 3 Appendix 3.1:

We have:

$$
\begin{aligned}
C_{1}^{1}\left(Q_{1}\right)=k . h \int_{x=L_{x}}^{U_{x}} \int_{Q_{A}=L_{Q A}}^{L_{x}}\left(x-Q_{A}\right) f(x) g\left(Q_{A}\right) d Q_{A} d x \\
C_{1}^{2}\left(Q_{1}\right)=h \int_{x=L_{x}}^{U_{Q_{A}}} \int_{U_{x}}^{U_{Q A}}\left(Q_{A}-x\right) f(x) g\left(Q_{A}\right) d Q_{A} d x \\
C_{1}^{3}\left(Q_{1}\right)=k \cdot h \int_{x=L_{x}}^{U_{Q_{A}}=L_{x}}\left(x-Q_{A}\right) f(x) g\left(Q_{A}\right) d Q_{A} d x \\
+h \int_{x=L_{x}}^{U_{x}} \int_{Q_{A}=x}^{U_{x}}\left(Q_{A}-x\right) f(x) g\left(Q_{A}\right) d Q_{A} d x
\end{aligned}
$$

The total cost function is written as the following:

$$
\begin{aligned}
C_{1}\left(Q_{1}\right) & =C_{1}^{1}\left(Q_{1}\right)+C_{1}^{2}\left(Q_{1}\right)+C_{1}^{3}\left(Q_{1}\right) \\
& =\frac{h\left(-6(k-1)\left(Q_{1}-\mu_{x}\right) \sigma_{\xi}+\sqrt{3}(k+1)\left(\left(-Q_{1}+\mu_{x}\right)^{2}+\sigma_{x}^{2}+3 \sigma_{\xi}^{2}\right)\right)}{12 \sigma_{\xi}}
\end{aligned}
$$

We can easily show that $C_{1}\left(Q_{1}\right)$ is convex, and by setting $\frac{\partial C_{1}\left(Q_{1}\right)}{\partial Q_{1}}=0$ and solving, we get $Q_{1}^{*}=\left[\mu_{x}+\sigma_{\xi} \sqrt{3} \frac{k-1}{k+1}\right]$

With an optimal cost function equal to: $C_{1}\left(Q_{1}^{*}\right)=\frac{h\left((k+1)^{2} \sigma_{x}^{2}+12 k \sigma_{\xi}^{2}\right)}{4 \sqrt{3}(k+1) \sigma_{\xi}}$.

Appendix 3.2:

The result obtained above is valid till:

1. If $k \geq 1$ we have $Q_{1}+\sqrt{3} \sigma_{\xi} \geq \mu_{x}+\sqrt{3} \sigma_{D}$ and $Q_{1}-\sqrt{3} \sigma_{\xi} \geq 0$. So by replacing $Q_{1}$ by $Q_{1}^{*}$ we get $\frac{k+1}{2} \sigma_{x} \leq \sigma_{\xi} \leq \frac{k+1}{\sqrt{12}} \mu_{x}$

2. If $k \leq 1$ we have $Q_{1}-\sqrt{3} \sigma_{\xi} \leq \mu_{x}-\sqrt{3} \sigma_{x}$ and $Q_{1}-\sqrt{3} \sigma_{\xi} \geq 0$. Again, by replacing $Q_{1}$ by $Q_{1}^{*}$ we get $\frac{k+1}{2 k} \sigma_{x} \leq \sigma_{\xi} \leq \frac{k+1}{\sqrt{12}} \mu_{x}$

For $k \geq 1$, it is easy to verify that $\frac{k+1}{2} \sigma_{x} \leq \frac{k+1}{\sqrt{12}} \mu_{x}$ since $L_{x}=\mu_{R}-\sqrt{3} \sigma_{x}$ is positive. $\frac{k+1}{2 k} \sigma_{x} \leq \frac{k+1}{\sqrt{12}} \mu_{x}$ is verified for values of $C V_{x}=\frac{\sigma_{x}}{\mu_{x}}$ such that $C V_{x} \leq \frac{k}{\sqrt{3}}$, otherwise Configuration 4 does not exist and the maximum value that can take $x_{x i}$ is between $\frac{2 k}{k+1}$ and $\frac{k+1}{2 k}$ (Configuration 2) is positive. 


\section{Appendix 4: Extension to the case with initial inventory}

In this Appendix, we consider the case of multiplicative errors with an initial inventory I. By following the methodology developed in this paper (cf Page 8), we extend our model and derive the optimal policy for each configuration.

If an initial inventory is taken into account, we show that that the ordering quantity in all configurations, except configuration 1 , is a non linear function of the initial inventory. The following result summarizes the overall optimal policy:

Result 7 For a given vector $\left(\mu_{x}, \sigma_{x}, k, I\right)$, we distinguish two cases: Case $A$ where $L_{Q_{A}} \leq L_{x}$ in the second configuration, i.e. $k \leq \frac{L_{x}+2 U_{x}-3 I}{2 L_{x}+U_{x}-3 I}$ and Case $B$ where $U_{Q_{A}} \geq U_{x}$ in the second configuration i.e. $k \geq \frac{L_{x}+2 U_{x}-3 I}{2 L_{x}+U_{x}-3 I}$. Depending on system parameters, in both cases, 1 ,2 or 3 of the configurations presented in Page 8 may be observed. The expression of the optimal quantity to order for each configuration as well as the critical values of $\sigma_{i j}$ can be determined by using the three steps approach described in Page 8:

\begin{tabular}{|l|l|l|}
\hline Conf. & $\begin{array}{l}\text { Interval of } \\
\sigma_{\gamma}\end{array}$ & $Q_{1}^{*}$ \\
\hline Conf. 1 & {$\left[0, \sigma_{12}\right]$} & $\frac{\mu_{\gamma}}{\mu_{\gamma}^{2}+\sigma_{\gamma}^{2}}\left[Q_{0}^{*}-I\right]$ \\
\hline Conf. 2 & {$\left[\sigma_{12}, \sigma_{23}\right]$} & $Q_{1}^{*}$ is obtained by solving $a Q_{1}^{* 3}+b Q_{1}^{* 2}+c=0$ \\
\hline Conf. 3 & {$\left[\sigma_{23}, \sigma_{\gamma \max }\right]$} & $\frac{\sqrt{(k+1)\left(\left(\mu_{x}-I\right)^{2}+\sigma_{x}^{2}\right)}}{\sqrt{-2 \sqrt{3}(k-1) \mu_{\gamma} \sigma_{\gamma}+(k+1)\left(\mu_{\gamma}^{2}+3 \sigma_{\gamma}^{2}\right)}}$ \\
\hline
\end{tabular}

\begin{tabular}{|l|l|}
\hline & $a=2(k+1) U_{\gamma}^{3}$ \\
Case A: $k \leq \frac{L_{x}+2 U_{x}-3 I}{2 L_{x}+U_{x}-3 I}$ & $b=3 k U_{x}\left(L_{\gamma}^{2}-U_{\gamma}^{2}\right)-3 L_{x}$ \\
$\sigma_{12}=\frac{\sqrt{\left(3\left(Q_{0}^{*}+I\right)-2 L_{x}\right)\left(2 L_{x}+Q_{0}^{*}+I\right)-16 I Q_{0}^{*}}-\sqrt{3}\left(Q_{0}^{*}-I\right)}{2\left(L_{x}-I\right)} \mu_{\gamma}$ & $\begin{array}{l}\left(U_{\gamma}^{2}+k L_{\gamma}^{2}\right)+3(k+1) I U_{\gamma}^{2} \\
c=(k+1)\left(L_{x}-I\right)^{3}\end{array}$ \\
$\sigma_{23}=\frac{(d-e)-\sqrt{(d-e)^{2}-e^{2}}}{\sqrt{3} e} \mu_{\gamma}$ & $d=k\left(U_{x}-I\right)^{2}$ \\
$\sigma_{\gamma \max }=\frac{\mu_{\gamma}}{\sqrt{3}}$ & $e=\left(\sqrt{3}\left(\mu_{x}-I\right)+\sigma_{x}\right)$ \\
& $(k+1) \sigma_{x}$ \\
\hline \hline & $a=2(k+1) L_{\gamma}^{3}$ \\
Case B: $k \geq \frac{L_{x}+2 U_{x}-3 I}{2 L_{x}+U_{x}-3 I}$ & $b=3 L_{x}\left(U_{\gamma}^{2}-L_{\gamma}^{2}\right)-3 U_{x}$ \\
$\sigma_{12}=\frac{\sqrt{3}\left(Q_{0}^{*}-I\right)-\sqrt{\left(3\left(Q_{0}^{*}+I\right)-2 U_{x}\right)\left(2 U_{x}+Q_{0}^{*}+I\right)-16 I Q_{0}^{*}}}{2\left(U_{x}-I\right)} \mu_{\gamma}$ & $\left(U_{\gamma}^{2}+k L_{\gamma}^{2}\right)-3 L_{\gamma}^{2}(k+1) I$ \\
$\sigma_{23}=\frac{(d+e)-\sqrt{(d+e)^{2}-e^{2}}}{\sqrt{3} e} \mu_{\gamma}$ & $c=(k+1)\left(U_{x}-I\right)^{3}$ \\
$\sigma_{\gamma \max }=\frac{\mu_{\gamma}}{\sqrt{3}}$ & $d=\left(L_{x}-I\right)^{2}$ \\
& $e=\left(\sqrt{3}\left(\mu_{x}-I\right)-\sigma_{x}\right)$ \\
& $(k+1) \sigma_{x}$ \\
\hline
\end{tabular}


For each critical value $\sigma_{i j}$ expressed in the table above, a condition on $I$ should be satisfied to assure $\sigma_{i j} \in R^{+}$and as a consequence, Configuration $j$ exists. This condition is expressed in the form of an interval of variation of $I$, as represented in the table below:

Case A : $k \leq \frac{L_{x}+2 U_{x}-3 I}{2 L_{x}+U_{x}-3 I}$

\begin{tabular}{|l|l|}
\hline Interval of $I$ & Possible conf. \\
\hline$\left[0, \mu_{x}-\sigma_{x} \frac{\sqrt{3}+\sqrt{(3-k)(k+1)}}{k}\right]$ & $1-2-3$ \\
\hline$\left[0, \frac{1}{3}\left(2 L_{x}+Q_{0}^{*}\right)\right]$ & $1-2$ \\
\hline$\left[0, Q_{0}^{*}\right]$ & 1 \\
\hline$\left[Q_{0}^{*},+\infty\right]$ & Do not order \\
\hline
\end{tabular}

Case B : $k \geq \frac{L_{x}+2 U_{x}-3 I}{2 L_{x}+U_{x}-3 I}$

\begin{tabular}{|l|l|}
\hline Interval of $I$ & Possible conf. \\
\hline$\left[0, L_{x}\right]$ & $1-2-3$ \\
\hline$\left[0,3 Q_{0}^{*}-2 U_{x}\right]$ & $1-2$ \\
\hline$\left[0, Q_{0}^{*}\right]$ & 1 \\
\hline$\left[Q_{0}^{*},+\infty\right]$ & Do not order \\
\hline
\end{tabular}

\section{Remarks:}

- By setting $I=0$ we find the results pertaining to the multiplicative errors case which are developed in this paper

- For Case A, an additional condition on model parameters should be made to ensure that the lower boundary of the received quantity reaches zero in Configuration 3 and as a consequence to ensure that Configuration 3 exists. This condition is as follows: $2-\frac{3 L_{x}\left(L_{x}+U_{x}\right)}{L_{x}^{2}+L_{x} U_{x}+U_{x}^{2}} \leq k \leq 3$

- For Case B, an additional condition should be made to ensure the existence of Configuration 2 and also to assure that $3 Q_{0}^{*}-2 U_{x} \geq 0$. This condition is as follows: $k \geq 2-3 \frac{L_{x}}{U_{x}}$

- For the particular case considered in Inderfurth (2004) $\left(L_{x}=0\right.$ and $L_{\gamma}=0$ ), our result confirm the optimal policy provided by the author:

$$
Q_{1}^{*}=\left\{\begin{array}{l}
\frac{1}{U_{\gamma}} \sqrt{\frac{\mu_{\gamma}}{3 U_{x}}} \text { if } I \in\left[0,3 Q_{0}^{*}-2 U_{x}\right] \\
\frac{\mu_{\gamma}^{2}+\sigma_{\gamma}^{2}}{\mu_{\gamma}^{2}} \text { if } I \in\left[3 Q_{0}^{*}-2 U_{x}, Q_{0}^{*}\right] \\
0 \text { if } I \in\left[Q_{0}^{*},+\infty\right]
\end{array}\right.
$$

